\title{
On approximation of asymmetric separators of the $n$-cube
}

\author{
Jan P Boroński ${ }^{1,2^{*}}$ and Marian Turzański ${ }^{3}$
}

\author{
* Correspondence: boronskij@mytu. \\ tuskegee.edu \\ ${ }^{1}$ Faculty of Applied Mathematics, \\ AGH University of Science and \\ Technology, al. Mickiewicza 30, 30- \\ 059 Kraków, Poland \\ Full list of author information is \\ available at the end of the article
}

\begin{abstract}
A new combinatorial result intertwined with the Brouwer fixed point theorem for the $n$ cube is given. This result can be used for any map $\left(f_{1}, \ldots, f n\right):[0,1]^{n} \rightarrow[0,1]^{n}$ to approximate the components of the set $\left\{\left(x_{1}, \ldots, x_{n}\right) \in[0,1]^{n}: f_{i}\left(x_{1}, \ldots, x_{n}\right)=x_{i}\right\}$ that separate the $n$-cube between the ith opposite faces. Equivalently, for maps $g:[0,1]^{n} \rightarrow$ $\mathbb{R}$ such that $g(x) g(y) \leq 0$ for any $x \in\{0\} \times[0,1]^{n-1}$ and $y \in\{1\} \times[0,1]^{n-1}$, one can use the algorithm to approximate the components of $g^{-1}(0)$ that separate $[0,1]^{n}$ between $\{0\} \times$ $[0,1]^{n-1}$ and $\{1\} \times[0,1]^{n-1}$. The methods are based on an earlier result of $P$. Minc and the present authors and relate to results of several other authors such as Jayawant and Wong, Kulpa and Turzański, and Gale.

Mathematics Subject Classification (2000): Primary 54H25; 54-04; Secondary 55M20; $54 \mathrm{~F} 55$.
\end{abstract}

Keywords: connected separators, algorithm, fixed point

\section{Introduction}

In [1] Minc and the present authors described combinatorial methods that allow approximation of connected symmetric separators of the $n$-sphere and $n$-cube. The symmetric separators arise in the context of the Borsuk-Ulam antipodal theorem and a theorem of Dyson for the 2-sphere [2,3]. The purpose of the present paper is to show how the results from [1] can be extended to the setting of asymmetric separators and the Brouwer fixed point theorem for the $n$-cube. The classic result of L.E.J. Brouwer says that the $n$-dimensional cube $I^{n}=[0,1]^{n}$ has the fixed point property; that is, for any $\operatorname{map}^{\mathrm{a}} f: I^{n} \rightarrow I^{n}$, there is $x \in I^{n}$ such that $f(x)=x$. There are many important applications of the Brouwer's theorem such as, for example, those concerning existence of solutions for differential equations [4], or equilibrium strategies in multi-person games relating to market problems in economics [5]. This is why computability of fixed points became an important theme in the fixed point theory. The first fixed point algorithm was given by Scarf [6]. Soon after, other were given by Eaves [7] and Todd [8] (see, for example, $[9,10]$ for a comprehensive treatment of this subject with applications). There are also several combinatorial equivalents of Brouwer's theorem. The best known is probably Sperner's lemma [11] on coloring vertices of a barycentric subdivision of an $n$-simplex. Some other transfer the fixed point problem to the scenario of board games, such as Hex [12] or Chess [13].

\section{Springer}

(c) 2012 Borońński and Turzańński; licensee Springer. This is an Open Access article distributed under the terms of the Creative Commons Attribution License (http://creativecommons.org/licenses/by/2.0), which permits unrestricted use, distribution, and reproduction in any medium, provided the original work is properly cited. 
In the present paper, in Theorem 3.1, we formulate yet another combinatorial result that implies the Brouwer fixed point theorem. Its baby version can be formulated as follows.

Theorem 1.1. Suppose $f: V \rightarrow \mathbb{R}$ is a function defined on the set of vertices $V$ of a triangulation $T$ of $I^{n}$. Suppose in addition that $f\left(v_{1}\right) f\left(v_{2}\right) \leq 0$ for any vertices $v_{1} \in\{0\} \times I^{n-1}$ and $v_{2} \in\{1\} \times I^{n-1}$. Then, there is a subcollection $S \subseteq T$ of simplices of dimension $n$ such that

1. for every simplex $\sigma \in S$ there is an edge $[v, u]$ such that $f(v) f(u) \leq 0$;

2. US separates $I^{n}$ between $\{0\} \times I^{n-1}$ and $\{1\} \times I^{n-1}$.

The above theorem implies the Brouwer fixed point theorem in the following way. If $\left(f_{1}, \ldots, f_{n}\right): I^{n} \rightarrow I^{n}$ is a map and $\mathcal{X}$ is a polyhedral complex with $I^{n}$ as its underlying space, then each $g_{i}\left(x_{1}, \ldots, x_{n}\right)=f_{i}\left(x_{1}, \ldots, x_{n}\right)-x_{i}$ satisfies the assumptions of Theorem 1.1 and one can find $C_{i}$, an approximation of a component of $g_{i}^{-1}(0)$, that separates $I^{n}$ between the $i$ th opposite $(n-1)$-dimensional faces. By Eilenberg-Otto theorem (see [14]) $\bigcap_{i=1}^{n} C_{i}$ is nonempty and approximates a fixed point of $f$.

We give a stronger (but at the same time more technical) version of the above result in Theorem 3.1, and in Section 4, we show how along with Theorem 4.1 it can be used to approximate a connected separating component of the set of zeros of an arbitrary map $f$ $: I^{n} \rightarrow \mathbb{R}$, which assumes opposite signs on some two opposite $(n-1)$-faces of $I^{n}$. The case when $n=2$ was already considered in [15]. The methods used in the proof of Theorem 3.1 are based on those introduced in [1] where, in connection with the BorsukUlam antipodal theorem, it was shown how to approximate a connected separator of the $n$-sphere $S^{n}$ (or $I^{n}$ ), invariant under the antipodal map. Any such separator was corresponding to a component of $f^{1}(0)$, with $f: S^{n} \rightarrow \mathbb{R}$ (or $f: I^{n} \rightarrow \mathbb{R}$ ) an odd map (related combinatorial results can be found in $[2,16])$. However, the methods of [1] were dealing only with symmetric separators and are insufficient in the case of arbitrary separators. First, unlike in the case of symmetric separators and odd maps, if a map $f: I^{n} \rightarrow$ R satisfies the condition $f\left(\{1\} \times I^{n-1}\right) \subseteq[0, \infty)$ and $f\left(\{0\} \times I^{n-1}\right) \subseteq(-\infty, 0]$ for some $i=1, \ldots, n$, there may be no unique connected separator of $I^{n}$ in $f^{1}(0)$. Clearly, $f^{1}(0)$ may consist of several disjoint separating components, none of which needs to be symmetric. Second, the algorithms in [1] were making use of the fact that the symmetric component of $f^{1}(0)$ is the separating omponent, when $f$ is odd. Therefore, if a subcollection of the triangulation approximated a component of $f^{1}(0)$ and, at the same time, was symmetric, this was sufficient to determine that it separated $S^{n}$ (or $I^{n}$ ). This is why one is forced to develop new combinatorial criteria for arbitrary separators in $I^{n}$. In Section 4 we furnish such a computer implementable criterion that allows isolating those subcollections of the triangulation, approximating a component of $f^{1}(0)$, that separate $I^{n}$ from those that do not.

\section{Preliminaries}

For a collection of sets $\mathcal{K}$, by $\mathcal{K}^{*}$ we will denote the union of all its elements. $\pi_{i}:[0,1]^{n} \rightarrow$ $[0,1]$ will denote the projection onto the $i$ th coordinate. $I_{i}^{+}$and $I_{i}^{-}$will denote the $i$ th opposite $(n-1)$-dimensional faces of $I^{n}$, that is $I_{i}^{+}=\pi_{i}^{-1}(1)$ and $I_{i}^{-}=\pi_{i}^{-1}(0)$. C separates $I^{n}$ (or is a separator of $I^{n}$ ) between $I_{i}^{+}$and $I_{i}^{-}$if for any $x \in I_{i}^{+} \backslash C, y \in I_{i}^{-} \backslash C$, there are $U, V$, distinct components of $I^{n} \backslash C$, such that $x \in U$ and $y \in V$. A map $g: \mathcal{X}^{*} \rightarrow \mathbb{R}$ is piecewise linear if given $\left\{\sigma_{j}: j=1, \ldots, N\right\}$, a triangulation of $\mathcal{X}^{*}$, for every $j$ the restriction of $g$ to 
the simplex $\sigma_{j}$ is linear, that is $g\left(\sum_{i=1}^{k} \lambda_{i} a_{i}\right)=\sum_{i=1}^{k} \lambda_{i} g\left(a_{i}\right)$ where $a_{1}, \ldots, a_{k}$ are the vertices spanning $\sigma_{j}$ and $\lambda_{i} \geq 0$ with $\sum_{i=1}^{k} \lambda_{i}=1$ (see [17]).

We will heavily rely on the following inductive procedure introduced by Minc and the two authors in [1]. Let $\mathcal{X}$ be a polyhedral complex such that $\mathcal{X}^{*}=[0,1]^{n}$. Let $\mathcal{V}(\mathcal{X})$ and $\mathcal{E}(\mathcal{X})$ denote the collections of vertices and edges, respectively. Suppose $f: \mathcal{V}(\mathcal{X}) \rightarrow \mathbb{R}$ is a function. Let $\mathcal{E}_{f}$ be the collection of those edges $e=\langle u, v\rangle \in \mathcal{E}(\mathcal{X})$ that $f(u) f(v) \leq 0$. Let $\mathcal{P} \subset \mathcal{X}$ be the collection of polytopes in $\mathcal{X}$ of dimension $n$. For any $e \in \mathcal{E}_{f} \mathcal{L}(e)$ is defined by induction.

- Let $\mathcal{C}_{0}(e)$ be the collection of those $P \in \mathcal{P}$ that contain $e$.

- Suppose $\mathcal{C}_{i-1}(e)$ has been defined. Define $\mathcal{C}_{i}(e)$ to be the collection of those $P \in \mathcal{P}$ such that the intersection $P \cap \mathcal{C}_{i-1}(e)^{*}$ contains an edge from $\mathcal{E}_{f}$ or a vertex from $f^{1}(0)$.

Clearly, $\mathcal{C}_{i-1}(e) \subset \mathcal{C}_{i}(e)$ and there is an integer $q \geq 0$ such that $\mathcal{C}_{q}(e)=\mathcal{C}_{q+1}(e)$. For the first such number $q(e)$ set $\mathcal{C}(e)=\mathcal{C}_{q(e)}(e)$. Note that $e \subset \mathcal{C}(e)^{*}$, and $\mathcal{C}(e)^{*}$ is connected.

\section{Combinatorial theorem on separators of $I^{\boldsymbol{n}}$ between opposite faces}

Let $\chi$ be a polyhedral complex such that $\mathcal{X}^{*}=[0,1]^{n}$. Note that $\mathcal{X}$ can be subdivided to give a triangulation of $I^{n}$, without introducing new vertices [18], and consequently, every function $f: \mathcal{V}(\mathcal{X}) \rightarrow \mathbb{R}$ has a piecewise linear extension $g: I^{n} \rightarrow \mathbb{R}$. The following result is of purely combinatorial nature.

Theorem 3.1. Suppose that $f: \mathcal{V}(\mathcal{X}) \rightarrow$ Ris a function satisfying

$$
f\left(\mathcal{V}(\mathcal{X}) \cap I_{i}^{+}\right) \subseteq[0,+\infty), \quad f\left(\mathcal{V}(\mathcal{X}) \cap I_{i}^{-}\right) \subseteq(-\infty, 0]
$$

for some $i \in\{1, \ldots, n\}$. Let $\mathcal{L}$ be a subcollection of $\mathcal{E}(\mathcal{X})$ such that $\mathcal{L}^{*} \subseteq I_{j}^{\varepsilon}$ for some $j \neq i$ and $\varepsilon \in\{+,-\}$, and $\mathcal{L}^{*} i$ s an arc with endpoints in $I_{i}^{+}$and $I_{i}^{-}$. Then, there is an edge $d \in \mathcal{E}(\mathcal{X}) \cap \mathcal{L}$ such that $d \in \mathcal{E}_{\text {f and }}$

1. each $C \in \mathcal{C}(d)$ contains an edge from $\mathcal{E}_{f}$,

2. $\mathcal{C}(d)^{*}$ separates $I^{n}$ between $I_{i}^{+}$and $I_{i}^{-}$and

3. for any other such $d^{\prime} \in \mathcal{L}$ with $\mathcal{C}\left(d^{\prime}\right)$ satisfying (1)-(2), either $\mathcal{C}\left(d^{\prime}\right) \cap \mathcal{C}(d)=\emptyset$ or $\mathcal{C}\left(d^{\prime}\right)=\mathcal{C}(d)$.

Proof. Without loss we can assume that $i=1$. Let $g: I^{n} \rightarrow \mathbb{R}$ be a piecewise linear extension of $f$. Then, $g$ is continuous and $g(v)=f(v)$ for any $v \in \mathcal{V}(\mathcal{X})$.

Claim 3.1.1. If $K \in \mathcal{X}$, then $g(r)=0$ for some $r \in K$ if and only if there is an edge $[w, v] \in \mathcal{E}(\mathcal{X})$ such that $w, v \in K \cap \mathcal{V}(\mathcal{X})$ and $f(w) f(v) \leq 0$.

Proof of claim 3.1.1. First suppose $K \in \mathcal{X}$ is such that there are vertices $w, v \in K \cap \mathcal{V}(\mathcal{X})$, and $f(w) f(v) \leq 0$. Then, either $f(w) f(v)=0$ or $f(w) f(v)<0$. In the first case, clearly $g(w)=f(w)=0$ or $g(v)=f(v)=0$. Otherwise there must be $r \in[u, v]$ such that $g(r)=0$. For the converse, suppose $g(r)=0$ for some $r \in K$. Then, $r=\sum_{i=1}^{k} \lambda_{i} a_{i}$, where $a_{o}, \ldots, a_{k}$ are vertices of $K$ spanning a simplex $\sigma \subseteq K$, and $\lambda_{i} \geq 0$ with 
$0=g(r)=\sum_{i=1}^{k} \lambda_{i} g\left(a_{i}\right)=\sum_{i=1}^{k} \lambda_{i} f\left(a_{i}\right)$. Therefore, $0=g(r)=\sum_{i=1}^{k} \lambda_{i} g\left(a_{i}\right)=\sum_{i=1}^{k} \lambda_{i} f\left(a_{i}\right)$. Clearly, there is $l$ such that $f\left(a_{l}\right)=0$, or there are $a_{j}, a_{t}$ such that $f\left(a_{j}\right) f\left(a_{t}\right)<0$.

Claim 3.1.2. $g\left(I_{i}^{+}\right) \subseteq[0,+\infty), g\left(I_{i}^{-}\right) \subseteq(-\infty, 0]$.

Proof of claim 3.1.2. Similarly to the proof of claim 3.1.1, this follows from the fact that if $a_{o}, \ldots, a_{k}$ spans a simplex $\sigma$ and $g\left(a_{i}\right) \geq 0\left(g\left(a_{i}\right) \leq 0\right)$ for every $i$, then $\sum_{i=1}^{k} \lambda_{i} g\left(a_{i}\right) \geq 0\left(\sum_{i=1}^{k} \lambda_{i} g\left(a_{i}\right) \leq 0\right)$ for $\lambda_{i} \geq 0$. Consequently, $g(\sigma) \subseteq[0,+\infty)(g(\sigma) \subseteq(-\infty$, $0])$ for any such $\sigma$.

Now, consider the following decomposition of the $n$-cube.

$Z=\left\{x \in I^{n}: g(x)=0\right\}, N=\left\{x \in I^{n}: g(x)<0\right\}, P=\left\{x \in I^{n}: g(x)>0\right\}$. Clearly $Z$ separates $I^{n}$ between $P$ and $N$. Let $Z_{1}, \ldots, Z_{p}$ be the components of $Z$. It is well known that if $X$ is a connected, locally connected and unicoherent space then any closed set separating $X$ contains a connected subset separating $X$ ([[19], p. 195], cf. [[20], p. 429, Theorem 1.(vi)]). Since $Z$ is closed and separates $I^{n}$, by unicoherence of $I^{n}$, there must be $q$ such that $Z_{q}$ separates $I^{n}$ between $N$ and $P$. Consequently, $Z_{q}$ separates $I^{n}$ between $I_{i}^{+}$and $I_{i}^{-}$by claim 3.1.2.

Consider $\mathcal{S}$ a subcollection of $\mathcal{K}$ such that $K \in \mathcal{S}$ if, it has nonempty intersection with $Z_{q}$, that is $\mathcal{S}=\left\{K \in \mathcal{X}: K \cap Z_{q} \neq \emptyset\right\}$. Clearly $Z_{q} \subseteq \mathcal{S}^{*}$ and therefore $\mathcal{S}^{*}$ separates $I^{n}$ between $I_{i}^{+}$and $I_{i}^{-}$.

Now, let $\mathcal{L}$ be a subcollection of $\mathcal{E}(\mathcal{X})$ such that $\mathcal{L}^{*} \subseteq I_{j}^{\varepsilon}$ for some $j \neq 1$ and $\varepsilon \in\{+,-\}$, and $\mathcal{L}^{*}$ is an arc with endpoints $a \in I_{1}^{+}$and $b \in I_{1}^{-}$, that is $\mathcal{L}^{*}=[a, b]$. Since $Z_{q} \cap \operatorname{Bd}\left(I^{n}\right)$ separates $\mathrm{Bd}\left(I^{n}\right)$ between $I_{i}^{+}$and $I_{i}^{-}$, we conclude there is $z \in[a, b] \cap Z_{q}$. Additionally, there is $d \in \mathcal{L}$ such that $z \in d$. By claim 3.1.1 $d \in \mathcal{E}_{f} \cap \mathcal{L}$, and since $d \cap Z_{q} \neq \varnothing$ therefore $d \in \mathcal{S}$.

Claim 3.1.3. $S \subseteq \mathcal{C}(d)$.

Proof of claim 3.1.3. Let $L \in \mathcal{S}$ be such that $d \in L$. Clearly $L \in \mathcal{C}_{0}(d) \subseteq \mathcal{C}(d)$. Heading toward a contradiction suppose $\mathcal{S} \backslash \mathcal{C}(d) \neq \emptyset$. Consider a partition of $\mathcal{S}$ into the following two sets

$$
\mathcal{S}_{1}=\{T \in \mathcal{S}: T \in \mathcal{C}(d)\}, \mathcal{S}_{2}=\{T \in \mathcal{S}: T \notin \mathcal{C}(d)\} .
$$

By definition of $\mathcal{C}(d)$, for any $T \in \mathcal{S}_{2}$ and for any $\tilde{T} \in \mathcal{S}_{1}$, we must have that whenever $s \in \mathcal{E}(\mathcal{X})$ and $s \subseteq T \cap \tilde{T}$ then $s \notin \mathcal{E}_{f}$. Otherwise $T$ would be in $\mathcal{S}_{1}$. Therefore, $(T \cap \tilde{T}) \cap Z_{q}=\emptyset$, by claim 3.1.1. Consequently, there is a partition of $Z_{q}$ into two disjoint sets $Z_{q} \cap \mathcal{S}_{1}^{*}$ and $Z_{q} \cap \mathcal{S}_{2}^{*}$. Since both are closed, we obtain a contradiction with connectedness of $Z_{q}$.

Now, property (1) is an immediate consequence of the definition of $\mathcal{C}(d)$. Since $\mathcal{S}^{*} \subseteq^{*} \mathcal{C}(d)$, (2) easily follows from the fact that $\mathcal{S}^{*}$ separates $I^{n}$ between $I_{i}^{+}$and $I_{i}^{-}$. Now, suppose $d^{\prime} \in \mathcal{L}$ is another edge with $\mathcal{C}\left(d^{\prime}\right)^{*}$ satisfying (1)-(2). If $\mathcal{C}\left(d^{\prime}\right) \cap \mathcal{C}(d) \neq \emptyset$, then there is $K$ such that $K \in \mathcal{C}_{j}(d)$ and $K \in \mathcal{C}_{p}\left(d^{\prime}\right)$ for some $j$ and $p$. Consequently, $\bigcup_{i=0}^{j} \mathcal{C}_{i}(d) \subseteq \bigcup_{i=p}^{q\left(d^{\prime}\right)} \mathcal{C}_{i}\left(d^{\prime}\right)$ and $\bigcup_{i=j}^{q(d)} \mathcal{C}_{i}(d) \subseteq \bigcup_{i=p}^{q\left(d^{\prime}\right)} \mathcal{C}_{i}\left(d^{\prime}\right)$, by definition of $\mathcal{C}\left(d^{\prime}\right)$. Clearly $\mathcal{C}(d) \subseteq \mathcal{C}\left(d^{\prime}\right)$. Similarly $\mathcal{C}\left(d^{\prime}\right) \subseteq \mathcal{C}(d)$. That justifies (3) and completes the proof. 


\section{Algorithm approximating connected separators of $I^{\mathbf{n}}$}

Suppose $\mathcal{K}$ is a partition of $I^{n}$ into $k^{n}$ congruent $n$-cubes, all with side length equal to $\frac{1}{k}$. In this section we shall furnish a computer implementable criterion for the union of a subcollection of $\mathcal{K}$ to separate $I^{n}$ between some two opposite faces. Suppose $\mathcal{S} \subseteq \mathcal{K}$ and we want to determine if $\mathcal{S}^{*}$ separates $I^{n}$ between $I_{i}^{+}$and $I_{i}^{-}$.

Let $K \in \mathcal{K} \backslash \mathcal{S}$ be an $n$-cube. $G$ is a $j$-face of $K$ if $\operatorname{dim}(G)=j$ and $G=K \cap L$ for some $K, L \in \mathcal{K}$. We will define Comp $(K, \mathcal{S})$ by induction. Let $\operatorname{Comp}_{1}(K, \mathcal{S})$ consists of $K$ and all those cubes $L$ in $\mathcal{K} \backslash \mathcal{S}$ such that $K \cap L$ is an $(n-1)$-face. Suppose $\operatorname{Comp}_{p}(K, \mathcal{S})$ has already been defined and let $\operatorname{Comp}_{p+1}(K, \mathcal{S})$ consists of all cubes in $\operatorname{Comp}_{p}(K, \mathcal{S})$, and all those cubes $R$ in $\mathcal{K} \backslash \mathcal{S}$ for which there is a cube $L \in \operatorname{Comp}_{p}(K, \mathcal{S})$ such that $L \cap$ $R$ is an $(n-1)$-face. Since $\mathcal{K}$ consists of only finite number of cubes $\operatorname{Comp}_{q}(K, \mathcal{S})=$ $\operatorname{Comp}_{q+1}(K, \mathcal{S})$ for some natural number $q$. Let $q(K)$ be the first such number and let $\operatorname{Comp}(K, \mathcal{S})=\operatorname{Comp}_{q(K)}(K, \mathcal{S})$.

Theorem 4.1. $\mathcal{S}^{*}$ separates $I^{n}$ between $I_{i}^{+}$and $I_{i}^{-}$iff

$$
\operatorname{Comp}(K, \mathcal{S})^{*} \cap I_{i}^{-}=\emptyset \text { for every } K \in \mathcal{K} \text { such that } K \cap I_{i}^{+} \neq \emptyset \text {. }
$$

Proof. If the condition (4.1) is not satisfied, then clearly $\mathcal{S}^{*}$ does not separate $I^{n}$ between $I_{i}^{+}$and $I_{i}^{-}$. Namely, Comp $(K, \mathcal{S})^{*}$ for some $K$ contains a connected set, disjoint with $\mathcal{S}^{*}$, intersecting both $I_{i}^{+}$and $I_{i}^{-}$in a nonempty set. For the converse, by contradiction suppose that the condition (4.1) is satisfied but $\mathcal{S}^{*}$ does not separate $I^{n}$ between $I_{i}^{+}$ and $I_{i}^{-}$. Let $A$ be a connected component of $I^{n} \backslash \mathcal{S}^{*}$ intersecting both $I_{i}^{+}$and $I_{i}^{-}$in a nonempty set. Let $\mathcal{R}$ be a subcollection of $\mathcal{K} \backslash \mathcal{S}$ such that $\mathcal{R}^{*}$ is connected and $\mathcal{A} \subseteq \mathcal{R}^{*}$. Without loss of generality, we can assume that $\mathcal{R}$ is a minimal such collection. We will obtain a contradiction showing, by induction, that for any two cubes in $\mathcal{R}$ if their intersection is an $m$-face, then $m \notin\{0, \ldots, n-2\}$. Suppose $K, L \in \mathcal{R}$ are two cubes such that $K \cap L \neq \varnothing$ but $L \notin \operatorname{Comp}(K, \mathcal{S})$. Since $K \cap L$ must be an $m$-face, for some $m<n$, we must have that $K \cap L$ is an $m$-face with $m<n-1$. Suppose $m=n$ - 2, then there are exactly $2^{2}-2$ other cubes sharing this $m$-face. Let $T$ be any of those two cubes. Then, $T \cap K$ and $T \cap L$ are $(n-1)$-faces, $K \cap L \subseteq T$ and $T$ must be in $\mathcal{S}$. A contradiction with the fact that $(K \cap L) \cap A \neq \varnothing$ and therefore $m \neq n-2$. Suppose we have already proved that $m<n-i$. We shall show that $m \neq n-(i+1)$. Suppose otherwise, that is $K \cap L$ is an $n-(i+1)$-face, for some $K, L \in \mathcal{R}$. Then, there are $2^{i+1}-2$ other cubes having this $n-(i+1)$-face in common. Let $T$ be one of them such that $T$ $\cap K$ is an $(n-1)$-face. Then, $T \cap L$ is an $(n-i)$-face and $K \cap L \subseteq T$. Since $(K \cap L) \subseteq T$ and $(K \cap L) \cap A \neq \varnothing$, therefore $A \cap T \neq \varnothing$ and $T \notin \mathcal{S}$. Consequently, $T \in \operatorname{Comp}(K, \mathcal{S})$ with $T \cap L$ an $(n-i)$-face, which leads to a contradiction by an inductive step.

It follows that for any two $K, L \in \mathcal{R}$ we have $L \in \operatorname{Comp}(K, \mathcal{S})$. Consequently, $\mathcal{R}=\operatorname{Comp}(K, \mathcal{S})$ for some $K$ such that $K \cap I_{i}^{+} \neq \emptyset$ and $\operatorname{Comp}(K, \mathcal{S})^{*} \cap I_{i}^{-} \neq \emptyset$. A contradiction that completes the proof.

A collection of cubes in $\mathcal{K}$ and the collection of the faces of all dimensions of cubes in $\mathcal{K}$ forms a polyhedral complex with $\mathcal{K}$ as its generating collection. Denote this complex by $\mathcal{X}$.

Suppose $f: \mathcal{V}(\mathcal{X}) \rightarrow \mathbb{R}$ is such that $f(v) \geq 0$ for each $v \in \mathcal{V}(\mathcal{X}) \cap I_{1}^{+}$and $f(v) \leq 0$ for each $v \in \mathcal{V}(\mathcal{X}) \cap I_{i}^{-}$. We will make use of Theorems 3.1 and 4.1 to obtain an algorithm finding $\mathcal{C}(d) \subset \mathcal{K}$, for all $d \in \mathcal{E}_{f}$, such that the following is true 
1. each $C \in \mathcal{C}(d)$ contains an edge from $\mathcal{E}_{f}$,

2. $\mathcal{C}(d)^{*}$ is connected, and

3. $\mathcal{C}(d)^{*}$ separates $I^{n}$ between $x \in I_{1}^{+}$and $y \in I_{1}^{-}$for each $x, y \in I^{n} \backslash \mathcal{C}(d)^{*}$.

Set $\mathcal{L}=\left\{\left[\frac{i}{k}, \frac{i+1}{k}\right] \times\{0\} \times \ldots \times\{0\}: i=0, \ldots, k-1\right\}$, and notice that $\mathcal{L}^{*}$ is a segment joining $I_{i}^{+}$and $I_{i}^{-}$. Therefore, $\mathcal{C}(d)$ will be the desired collection satisfying (1) - (3) for some $d \in \mathcal{L}$.

\section{Algorithm (outline)}

Step 1. Add all elements of $\mathcal{E}_{f} \cap \mathcal{L}$ to List A.

Step 2. Repeat Step 3-Step 11 until List A is empty.

Step 3. Pick an edge $d$ from List A.

Step 4. Generate $\mathcal{C}(d)$. Remove $d$ from List A.

Step 5. Add all elements $K \in \mathcal{K}$ such that $K \cap I_{i}^{+} \neq \emptyset$ to List B.

Step 6. Repeat Step 7-Step 9 until List B is empty.

Step 7. Pick a cube $K$ from List B.

Step 8. Generate Comp $(K, \mathcal{C}(d))$. Remove $K$ from List B.

Step 9. If there is $L \in \operatorname{Comp}(K, \mathcal{C}(d))$ such that $L \cap I_{i}^{-} \neq \varnothing \emptyset$ then go back to Step 3. Otherwise, go back to Step 7.

Step 10. List all elements from $\mathcal{C}(d)\left(\mathcal{C}(d)^{*}\right.$ is a separator).

Step 11. Go back to Step 3.

\section{Endnote}

${ }^{a}$ By a map we will always mean a continuous function. Whenever continuity is not assumed we will use the term function instead.

Acknowledgements

The authors are grateful to the referees for their careful reading of our manuscript and helpful comments that improved the paper.

\section{Author details}

${ }^{1}$ Faculty of Applied Mathematics, AGH University of Science and Technology, al. Mickiewicza 30, 30-059 Kraków, Poland ${ }^{2}$ Department of Mathematics, Tuskegee University, Tuskegee, AL 36088, USA ${ }^{3}$ Faculty of Mathematics and Natural Sciences, College of Sciences, Cardinal Stefan Wyszyński University, ul. Dewajtis 5, 01-815 Warszawa, Poland

\section{Authors' contributions}

Both the authors contributed equally to writing of the present paper. They also read and approved the final manuscript.

\section{Competing interests}

The authors declare that they have no competing interests.

Received: 1 February 2011 Accepted: 4 January 2012 Published: 4 January 2012

\section{References}

1. Boroński, JP, Minc, P, Turzański, M: Algorithms for finding connected separators between antipodal points. Topol Appl. 154(18), 3156-3166 (2007). doi:10.1016/j.topol.2007.08.013

2. Jayawant, P, Wong, P: A combinatorial analog of a theorem of F.J. Dyson. Topol Appl. 157(10-11), 1833-1838 (2010). doi:10.1016/j.topol.2010.02.020

3. Krasinkiewicz, J: Functions defined on spheres-remarks on a paper by K. Zarankiewicz. Bull Polish Acad Sci Math. 49(3), 229-242 (2001)

4. Lamberto, C: Asymptotic Behavior And Stability Problems In Ordinary Differential Equations. Ergebnisse der Mathematik und ihrer Grenzgebiete NF, Band 16. Springer, Berlin (1959)

5. Nash, J: Non-cooperative games. Ann Math. 54(2), 286-295 (1951). doi:10.2307/1969529

6. Scarf, H: The approximation of fixed points of a continuous mapping. SIAM J Appl Math. 15, 1328-1343 (1967). doi:10.1137/0115116

7. Eaves, BC: Homotopies for computation of fixed points. Math Program. 3, 1-22 (1972). doi:10.1007/BF01584975 
8. Todd, MJ: The computation of fixed points and applications. In Lecture Notes in Economics and Mathematical Systems, vol. 124,Springer, Berlin (1976)

9. Scarf, H: The computation of economic equilibria. With the collaboration of Terje Hansen. Cowles Foundation Monograph No 24. Yale University Press, New Haven, Conn.-London (1973)

10. Kojima, M: An introduction to variable dimension algorithms for solving systems of equations. Numerical solution of nonlinear equations (Bremen, 1980). In Lecture Notes in Mathematics, vol. 878, pp. 199-237.Springer, Berlin (1981). doi:10.1007/BFb0090683

11. Sperner, E: Neuer Beweis für die Invarianz der Dimensionzahl und des Gebieties. Abh Math Sem Ham Univ. 6, 265-272 (1928). doi:10.1007/BF02940617

12. Gale, D: The game of hex and the Brouwer fixed-point theorem. Am Math Mon. 86, 818-827 (1979). doi:10.2307/ 2320146

13. Tkacz, P, Turzański, M: A n-dimensional version of Steinhaus chessboard theorem. Topol Appl. 155(4), 354-361 (2008). doi:10.1016/j.topol.2007.07.005

14. Hurewicz, W, Wallman, H: Dimension Theory. Princeton University Press, Princeton (1948)

15. Turza®ski, M: Equlibrum theorem as the consequence of the Steinhaus chessboard theorem. Topol Proc. 25, 645-653 (2000)

16. Kulpa, W, Turzański, M: A combinatorial theorem for a symmetric triangulation of the sphere $S^{2}$. Acta Univ Carol Math Phys. 42(2), 69-74 (2001)

17. Rourke, CP, Sanderson, BJ: Introduction to piecewise-linear topology. Ergebnisse der Mathematik und ihrer Grenzgebiete, Band 69. Springer, New York (1972)

18. Aleksandrov, PS: Combinatorial Topology. Graylock, Rochester (1956)

19. Hunt, JHV: A characterization of unicoherence in terms of separating open sets. Fund Math. 107, 195-199 (1980)

20. Stone, AH: Incidence relations in unicoherent spaces. Trans Am Math Soc. 65, 427-447 (1949). doi:10.1090/S0002-99471949-0030743-8

doi:10.1186/1687-1812-2012-2

Cite this article as: Boroński and Turzański: On approximation of asymmetric separators of the $n$-cube. Fixed Point Theory and Applications 2012 2012:2.

\section{Submit your manuscript to a SpringerOpen ${ }^{\odot}$} journal and benefit from:

- Convenient online submission

- Rigorous peer review

- Immediate publication on acceptance

- Open access: articles freely available online

- High visibility within the field

- Retaining the copyright to your article

Submit your next manuscript at $\gg$ springeropen.com 